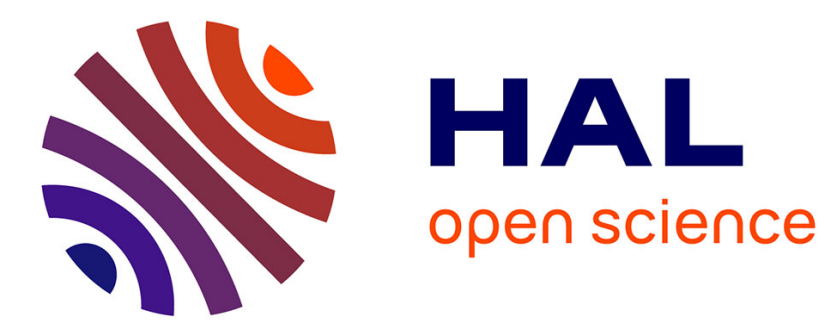

\title{
Treatment Discontinuation Following Bariatric Surgery in Obstructive Sleep Apnea: a Controlled Cohort Study
}

Claire Agosta, Jean-Christian Borel, Fabian Reche, Catherine Arvieux, Nelly Wion, Samir Jaber, Dany Jaffuel, Jean-Louis Pépin, Anne-Laure Borel

\section{- To cite this version:}

Claire Agosta, Jean-Christian Borel, Fabian Reche, Catherine Arvieux, Nelly Wion, et al.. Treatment Discontinuation Following Bariatric Surgery in Obstructive Sleep Apnea: a Controlled Cohort Study. Obesity Surgery, 2016, 26 (9), pp.2082 - 2088. 10.1007/s11695-016-2048-9 . hal-01792488

\section{HAL Id: hal-01792488 \\ https://hal.umontpellier.fr/hal-01792488}

Submitted on 22 Nov 2019

HAL is a multi-disciplinary open access archive for the deposit and dissemination of scientific research documents, whether they are published or not. The documents may come from teaching and research institutions in France or abroad, or from public or private research centers.
L'archive ouverte pluridisciplinaire HAL, est destinée au dépôt et à la diffusion de documents scientifiques de niveau recherche, publiés ou non, émanant des établissements d'enseignement et de recherche français ou étrangers, des laboratoires publics ou privés. 


\section{Treatment Discontinuation Following Bariatric Surgery in Obstructive Sleep Apnea: a Controlled Cohort Study}

Claire Agostal \& Jean-Christian Borel2,3 \& Fabian Reche4 \& Catherine Arvieux4 \& Nelly Wion2 \& Samir Jaber5 \& Dany Jaffuel6 \& Jean-Louis Pépin3,7 \& Anne-Laure Borel1,3

* Anne-Laure Borel alborel@chu-grenoble.fr

1 Pole DIGIDUNE, Endocrinology Department, Grenoble University Hospital, Grenoble, France

2 BICàdom^, Agiràdom, Meylan, France

3 HP2 laboratory, INSERM U1042, Grenoble Alpes University, Grenoble, France

4 Pole DIGIDUNE, Digestive Surgery Department, Grenoble University Hospital, Grenoble, France

5 Anaesthesiology and Intensive Care, Anaesthesia and Critical Care Department B, Saint Eloi Teaching Hospital, INSERM U1046, Montpellier 1 University, Montpellier University Hospital, Montpellier 34295, France

6 Pulmonary Disorders and Respiratory Sleep Disorders Unit, Polyclinic Saint-Privat, 34760 Boujan sur Libron, France

7 Pole Thorax et vaisseaux, Clinique Physiologie, Sommeil et Exercice, Grenoble University Hospital, Grenoble, France

Keywords Bariatric surgery . Sleep apnea syndrome .

Continuous positive airway pressure. Cohort. Controlled

\section{Abstract}

Background Uncontrolled studies looking at the discontinuation of obstructive sleep apnea (OSA) treatment after bariatric surgery (BS) have suggested that surgery improves OSA. However, this discontinuation of OSA treatment by BS patients has never been compared to a matched population without BS. The objectives of this study are to evaluate whether BS increases OSA treatment discontinuation compared to that in matched patients without BS and to identify predictive factors of OSA treatment discontinuation in BS patients. The study took place in an ambulatory, tertiary hospital.

Methods We included 61 OSA patients who underwent BS in a retrospective controlled cohort study. The computerized matching procedure included age, sex, body mass index, year of starting OSA treatment, treatment type, and duration selected 59 controls matched to 28 patients with BS. 
The main outcome was OSA treatment discontinuation within 2 years after BS .

Results Patients with BS stopped OSA treatment more often than controls, usually between 6 months and 1 year after BS: hazards ratio (HR (95 \%, CI)) 15.93 (3.29, 77.00). Before 6 months or beyond 1 year after BS, treatment discontinuation was not different between BS patients and controls. In univariate analyses, female gender, absence of co-morbidities, greater weight loss, and lower baseline OSA severity were associated with stopping OSA treatment after BS. No factor remained independently associated in multivariate analysis.

Conclusions Apneic patients having BS stop OSA treatment more than matched controls. Treatment discontinuation may be attributed to recovery or to abandonment. The effect of BS on OSA may have been overestimated in uncontrolled BS studies that ignored basal OSA treatment discontinuation in routine clinical practice.

\section{Introduction}

Obesity is a recognized risk factor for obstructive sleep apnea

(OSA) [1, 2]. OSA is characterized by repetitive episodes of partial or complete pharyngeal collapse during sleep-inducing intermittent hypoxia and sleep fragmentation. It is associated with excessive daytime sleepiness, an increase in the risk of road traffic accidents, deterioration in quality of life, and long-term cardiovascular morbidity and mortality. Continuous positive airway pressure (CPAP) is the first-line therapy for moderate-to-severe OSA. Both randomized controlled studies and observational cohort studies have demonstrated that CPAP is beneficial in terms of daytime vigilance and quality of life while its impact on cardiovascular mortality remains debated [36]. However, $15 \%$ of patients who present with severe OSA refuse to initiate positive airway pressure therapy and 20 to 30 stop this therapy during long-term follow-up [7-10]. OSA remains under-diagnosed among candidates for bariatric surgery [11, 12]. However, in this population, the prevalence of moderate-to-severe OSA ranges from 40 to $86 \div$ [13-16]. OSA seems to be drastically improved after bariatric surgery [17, 18]. Body weight loss is associated with a reduction in the apnea-hypopnea index, and the greater the reduction in body weight, the better the OSA improvement [19]. Two meta-analyses of studies including patients undergoing bariatric surgery suggest a remission of OSA in 80 o of cases [20, 21]. However, criteria used to define the resolution of OSA are questionable, often based on self-reported discontinuation of treatment. In fact, more than half the patients present with mild-to-moderate residual OSA when their apnea-hypopnea index is objectively assessed by polysomnography after surgery [22, 23]. Indeed, treatment discontinuation can either be understood as abandonment, or as actual recovery. 
Thus, OSA resolution after bariatric surgery when evaluated by CPAP discontinuation could be confounded for the abandonment of CPAP therapy often seen in routine clinical practice.

The main objective of the present study was to evaluate in obese OSA patients treated with positive airway pressure whether having bariatric surgery was associated with a higher rate of OSA treatment discontinuation compared to matched obese OSA patients who did not undergo bariatric surgery and whether the rate of OSA treatment discontinuation was specifically related to bariatric surgery, either by OSA recovery after weight loss or by increased abandonment. The secondary objective was to determine the factors predictive of patients stopping OSA treatment after bariatric surgery.

\section{Patients and Methods}

\section{Study Design}

This was a retrospective monocentric controlled cohort study.

Obese patients suffering from OSA and treated by nocturnal positive airway pressure (CPAP or bi-level positive airway pressure) who had undergone bariatric surgery were identified in the database of a 600 bed University Hospital (Grenoble, France). All bariatric surgery patients were followed for their positive airway pressure treatment with standardized procedures for treatment initiation and home followup by the same regional home-care provider ("AGIR à dom"). Controls were obese OSA patients treated with nocturnal positive airway pressure but without bariatric surgery followed in the database of the same regional home care provider. In line with French guidelines, OSA treatment was initiated in patients having severe OSA (apnea-hypopnea index $>30$ events/h) diagnosed by either polygraphy or polysomnography, daytime sleepiness, and at least three clinical symptoms of OSA.

Operated and control patients were carefully matched using the following criteria: same sex, modality of positive airway pressure therapy (CPAP or bi-level positive airway pressure), and treatment duration before surgery (i.e., matched controls had similar duration of treatment to that of operated patients before surgery), then age ( \pm 5 years), body mass index (BMI, $\pm 1 \mathrm{~kg} / \mathrm{m} 2$ ), and year of starting positive airway pressure treatment ( \pm 2.5 years). Matching (1 to 3 control patients per operated patient) was automatically performed using SAS® (version 9.2, OSA Institute, Cary, NC, USA).

\section{Main Outcome}

All discontinuations of positive airway pressure treatment during the 2 years following bariatric surgery and those during the 2 years following the start point in matched control patients were recorded. In matched controls, the start point was defined as the time when treatment duration had been the same as in operated patients at the time of surgery. 


\section{Secondary Outcomes}

In patients with bariatric surgery, potential predictive factors for stopping OSA treatment after surgery were tested: age, gender, BMI before surgery, medical history of cardiovascular disease (myocardial infarction, angina, stroke, and/or hypertension), medical history of metabolic disease (diabetes and/or dyslipidemia), medical history of respiratory disease (asthma, chronic obstructive pulmonary disease, and/or obesity hypoventilation syndrome), and percent of body weight loss 6 months after surgery.

\section{Statistical Analysis}

Results are expressed as means (standard deviation) for normally distributed variables and as median (interquartile range) for variables that did not show normal distribution. Categorical variables are presented as $\mathrm{n}\left(\frac{\circ}{0}\right)$. The normal distribution of residuals was verified by the Shapiro-Wilk test.

Descriptive Analyses For the variables that were not exactly the same after the matching procedure (i.e., age, BMI, and AHI), potential differences between operated patients and matched controls were tested by evaluating whether the mean of intra-strata differences was different from 0 (mixed model).

Main Outcome A marginal Cox model was built to determine the rate of stopping OSA treatment in operated patients vs. controls. The proportional hazard assumption was not respected. The main outcome was then tested: (i) from start point to 6 months after bariatric surgery, (ii) from 6 months to 1 year after bariatric surgery, and (iii) from 1 to 2 years after bariatric surgery.

Secondary Outcomes In patients referred to bariatric surgery, univariate Cox models were performed to look for predictive factors of positive airway pressure treatment discontinuation. A multivariable Cox model was then built, entering all variables that were associated with stopping OSA treatment in the univariate analysis having a $p$ value $<0.2$.

Comparisons with $\mathrm{p}<0.05$ were considered as significant. All analyses were performed using SAS version 9.2 (OSA Institute, Cary, NC, USA).

\section{Results}

Table 1 shows the characteristics of the 61 obese OSA patients treated by nocturnal positive airway pressure who had bariatric surgery and who were included in the matching procedure.

Table 1 Baseline characteristics of patients who underwent bariatric surgery 


\begin{tabular}{|c|c|}
\hline Patients referred to bariatric surgery & $(\mathrm{n}=61)$ \\
\hline Age (years) & $44 \quad(10)$ \\
\hline Sex (male) & $21 \quad(34.4)$ \\
\hline BMI $(\mathrm{kg} / \mathrm{m} 2)$ & $48.7 \quad(8.2)$ \\
\hline HI (events/h) & $47.1(35.8)$ \\
\hline OSA treatment duration (months) & $14(6-54)$ \\
\hline $\begin{array}{l}\text { Type of positive airway pressure therapy CPAP } \\
\text { BIPAP }\end{array}$ & $\begin{array}{l}54(88.5) \\
6(9.8)\end{array}$ \\
\hline BIPAP + oxygen & $1(1.6)$ \\
\hline Bariatric surgery Gastric banding & $5(8.2)$ \\
\hline Bypass & $34(55.7)$ \\
\hline Sleeve gastrectomy & $22(36.1)$ \\
\hline Body weight loss at 6 months ( $\frac{\circ}{0}$ ) & $-17.8(7.3)$ \\
\hline Body weight loss at 1 year ( $\left(\frac{0}{0}\right)$ & $-23.2(11.0)$ \\
\hline Body weight loss at 2 years $\left(\frac{\circ}{0}\right)$ & $-22.4(16.5)$ \\
\hline Medical history Cardiovascular disease & $31(50.8)$ \\
\hline Metabolic disease & $(67.2)$ \\
\hline Other respiratory disease & $(32.8)$ \\
\hline
\end{tabular}

BMI body mass index, AHI apnea-hypopnea index, OSA obstructive sleep apnea, CPAP continuous positive airway pressure, BIPAP bilevel positive airway pressure

The automatic procedure matched 59 "control" apneic patients with obesity with 28 OSA patients who had bariatric surgery. A flow chart of the study is presented in Fig. 1. The start point characteristics of patients selected by the matching procedure are reported in Table 2. The 33 operated patients who were not retained by the automatic matching procedure were not-statistically different from the 28

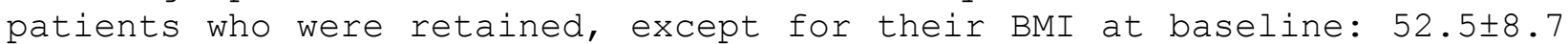
vs. $44.2 \pm 4.7 \mathrm{~kg} / \mathrm{m} 2$, respectively, $\mathrm{p}<0.001$.

\section{Main Outcome}

The percentage of patients who pursued nocturnal positive airway pressure therapy after the start point (i.e., bariatric surgery for operated patients, same treatment duration for controls) is shown in Fig. 2a (Kaplan-Meier representation). Since the proportional hazards assumption was not respected, i.e., the rate of OSA treatment discontinuation was not steady along the 2 years of the follow-up period, the rate of positive airway pressure therapy discontinuation was then compared from starting point to 6 months after starting point (Fig. 2b), from 6 months to 1 year (Fig. 2c), and from 1 to 2 years (Fig. 2d). The hazard ratios (95 \%, CI) were $1.11(0.33,3.75)$ from starting point to 6 months $(\mathrm{p}=0.864), 15.93(3.29,77.00)$ from 6 months to 1 year $(p<0.001)$, and $8.33(0.95,73.25)$ from 1 to 2 years of follow-up $(p=0.056)$.

\section{Secondary Outcomes}


Table 3 reports factors associated with the discontinuation of OSA treatment in all patients $(\mathrm{n}=61)$ that benefited from bariatric surgery in univariate Cox analyses. Patients were more likely to stop nocturnal positive airway pressure therapy after surgery if they were female, free of cardiovascular disease, if they had lost more than $18.4 \%$ of body weight at 6 months after surgery, and exhibited lower OSA severity at baseline $(<35$ events/h). In multiple Cox analysis, no variable remained independently associated with stopping OSA treatment after bariatric surgery.

\section{Discussion}

In this controlled cohort study, apneic patients with obesity referred to bariatric surgery demonstrated a higher rate of nocturnal positive airway pressure therapy discontinuation than carefully matched control apneic patients. This result indicates that the effect of bariatric surgery on treatment discontinuation was independent of the usual abandonment seen in control patients in routine clinical practice. Nevertheless, the specific effect of bariatric surgery may be related not only to OSA recovery, but also to an increased abandonment of OSA treatment in operated patients, related to the relief of symptoms, despite persistent sleep apnea.

Fig. 1 Flow chart

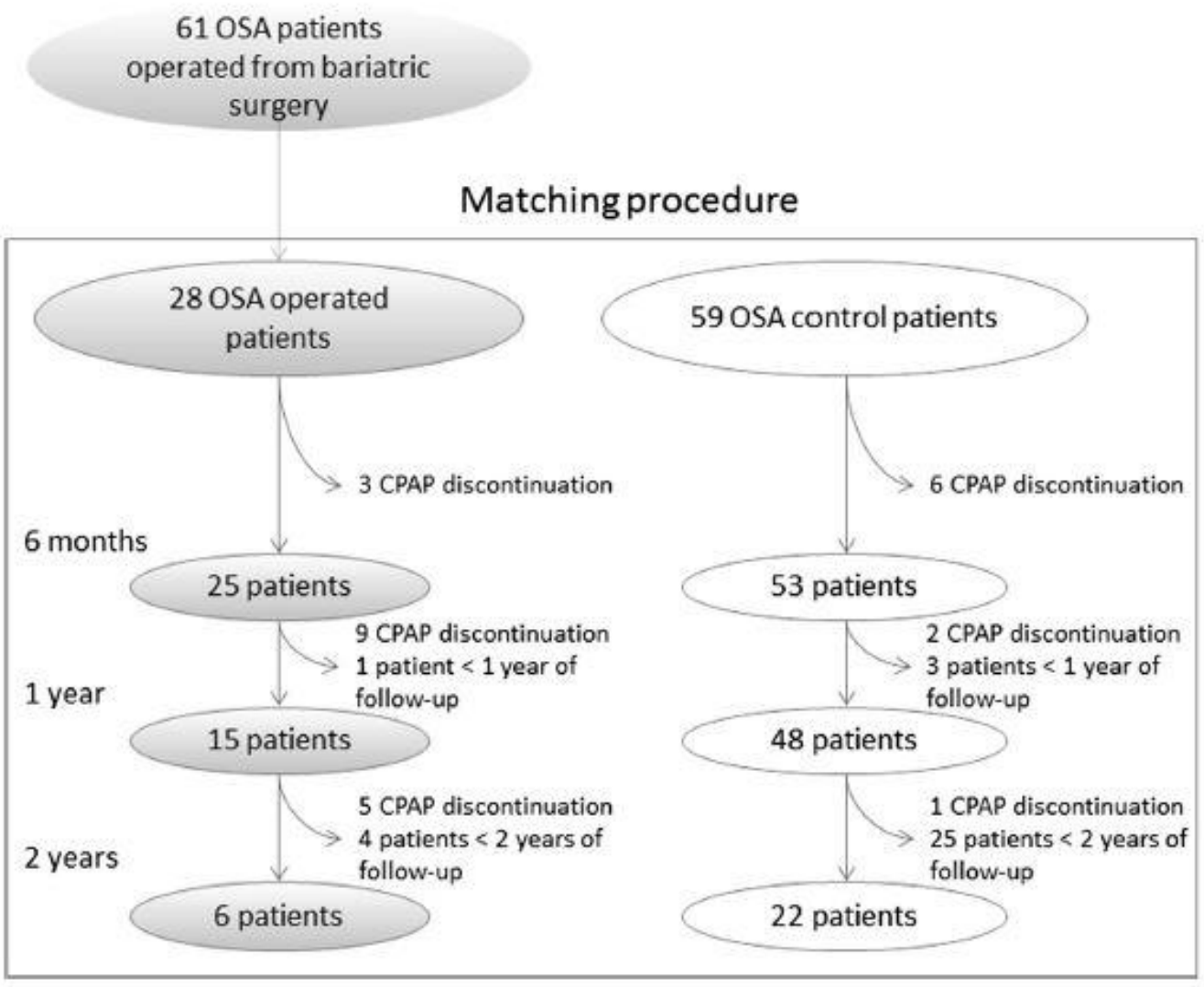


Various different factors are likely to influence positive airway pressure therapy adherence, including the patients'perception of symptom relief (less daytime sleepiness and better every day functioning), and also psychological and sociodemographic features (marital and employment status), and technical aspects [24]. Moreover, patient education and quality of follow-up play a major role in determining patterns of use [25-27].

Studies addressing OSA resolution or improvement after bariatric surgery have shown conflicting results. The meta-analysis by Buchwald et al. [20] included studies that assessed post-surgical OSA improvement or resolution using either subjective (self-reported cessation of OSA treatment) or objective (post-surgical polysomnography) outcomes. The

Table 2 Characteristics of patients selected by the matching procedure

$\begin{array}{cc}\text { OSA and bariatric } & \text { OSA without } \\ \text { surgery }(\mathrm{n}=28) & \text { bariatric surgery } \\ (\mathrm{n}=59) & \end{array}$

\begin{tabular}{|c|c|c|}
\hline Age (years) & $45 \quad(9)$ & $47(9)$ \\
\hline Sex (male) & $7(25.0)$ & $14(24.0)$ \\
\hline $\operatorname{BMI} \quad(\mathrm{kg} / \mathrm{m} 2)$ & $44.2(4.7)$ & $43.4 \quad(4.6)$ \\
\hline AHI (events/h) & $41.2(31.0)$ & $51.5 \quad(28.4$ \\
\hline $\begin{array}{l}\text { OSA treatment } \\
\text { duration (months) }\end{array}$ & $14(6-54)$ & $8(4-30)$ \\
\hline Type of positive & airway press & erapy \\
\hline CPAP & $26 \quad(92.9)$ & $57(96.6)$ \\
\hline BIPAP & $(7.1)$ & $(3.4)$ \\
\hline BIPAP + oxygen & $(0.0)$ & $(0.0)$ \\
\hline
\end{tabular}

For the variables that were not exactly similar after the matching procedure (i.e., age, BMI, and AHI), potential differences between operated patients and matched controls were tested by evaluating whether the mean intra-strata difference was different from 0 (mixed model). There were no intra-strata differences for sex or treatment duration at starting point that were by definition identical according to matching procedure

BMI body mass index, AHI apnea-hypopnea index, OSA obstructive sleep apnea, CPAP continuous positive airway pressure, BIPAP bi-level positive airway pressure 

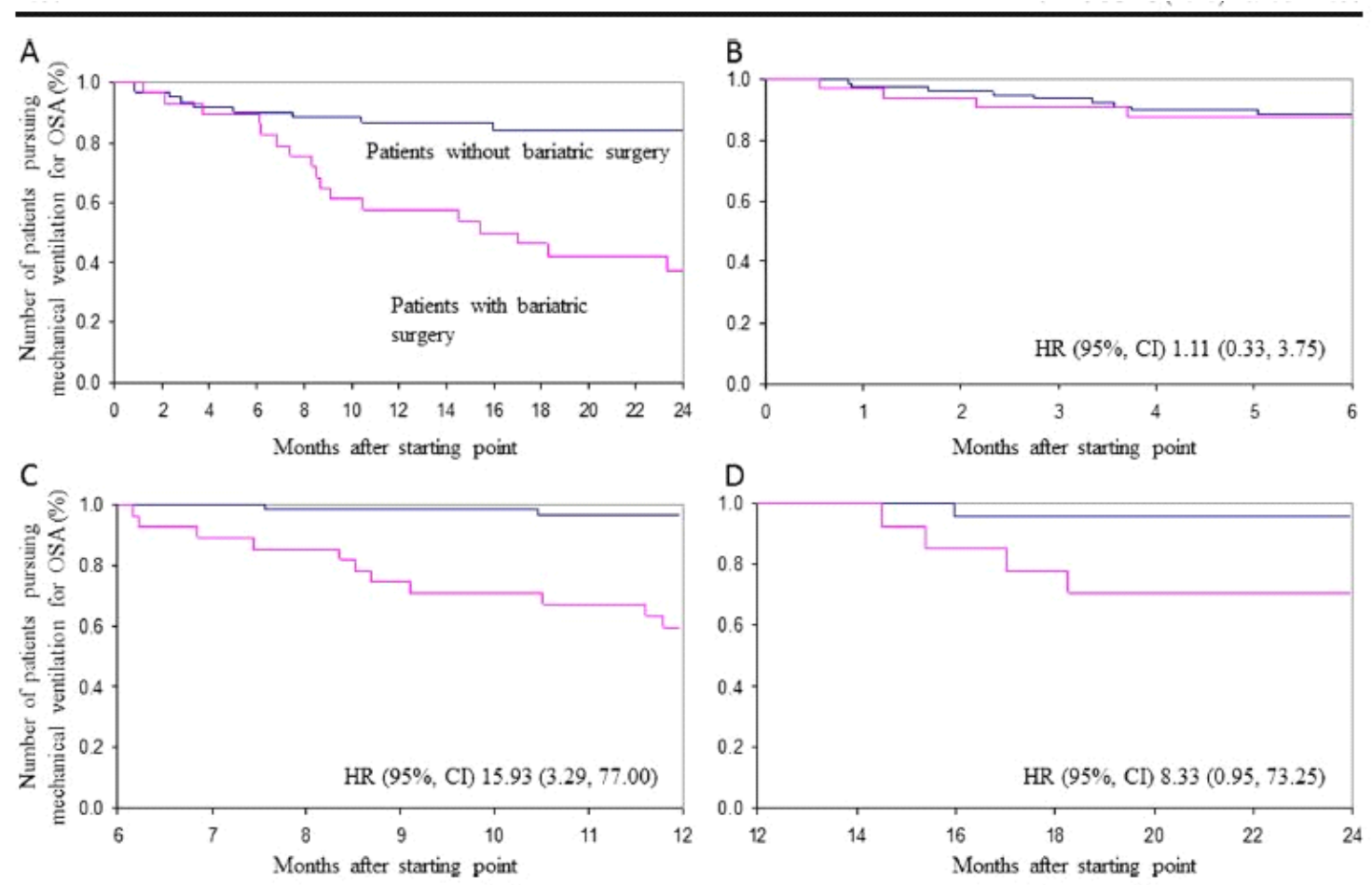

Fig. 2 Kaplan-Meier curves of the percentage of patients who pursued mechanical ventilation for obstructive sleep apnea (OSA).

a Kaplan-Meier curves of operated-patients and controls between 6 months and Meier curves of patients with bariatric surgery (28 operated patients) and patients without bariatric surgery (59 controls) between start point and 2 years after start point. b Kaplan-Meier curves of operated patients and controls between start point and 6 months after start point. C Kaplan-Meier curves of operated-patients and controls between 6 months and 1 year after start point. d Kaplan-Meier curves of operated patients and controls between 1 and 2 years after start point.

The authors found that OSA resolved in $85.7 \div(95 \div \mathrm{CI}, 79.2$ - after bariatric surgery, indicating the need to pursue CPAP 92.2 o) of OSA patients. Greenburg et al. [22] limited their therapy or to propose alternative treatments. This observation analysis to the 12 studies in which an apnea-hypopnea index led the authors to warn health-care providers that the benefits (AHI) was objectively measured by polysomnography before of bariatric weight loss may lead patients to subjectively feel and after bariatric surgery. They found significant residual well despite the persistence of significant OSA. Based on this OSA (AHI $>15$ events/h) in the majority of patients (62 \%) meta analysis, one could expect 38 of patients to discontinue CPAP therapy after bariatric surgery, based on a reduction of AHI to <15 events/h.

Table 3 Predictive factors for stopping positive airway pressure therapy for obstructive sleep apnea in patients referred tobariatric surgery 
Age above vs. below

the median (44.5)

Sex (female vs. male)

BMI above vs. below

the median $(47.4 \mathrm{~kg} / \mathrm{m} 2)$

Medical history of CVD

Medical history of

type 2 diabetes

Medical history of

respiratory disease

Percent of body weight

loss at 1 month: above vs.

below the median $(-8.8 \%)$

Percent of body weight

loss at 6 months: above vs.

below the median $(-18.4 \%)$

AHI above vs. below the

median (35 events/h)
$0.825(0.34,2.03)$
0.676
0.029
$0.89(0.36,2.19)$
0.796
$0.33(0.12,0.92)$
0.033
$0.75(0.30,1.91)$
0.545
$0.53(0.18,1.59)$
0.260
$0.62(0.25,1.54)$
0.303
$0.31(0.10,0.96)$
0.044
$0.29(0.10-0.82)$
0.019

Associations were tested by univariate Cox analyses BMI body mass index, CVD cardiovascular disease, HR hazard ratio, AHI apnea hypopnea index

In the present study, the extent of discontinuation (61 \%) was $23 \%$ higher. The estimation of $23 \%$ abandonment due to bariatric surgery, without OSA resolution, exceeds the $15 \%$ dropout observed in the control group. This suggests that bariatric surgery is indeed a chance to recover from OSA but also increases the risk of inappropriate discontinuation of OSA treatment. This might be because of the relief from symptoms or because patients subjectively overestimate the impact of body weight reduction.

This is in agreement with a recent report that 1 year after bariatric surgery CPAP adherence drops from $83.3 \%$ preoperatively to $38.1 \%$ even when OSA persisted [28].

In the matched control group, 15 of apneic patients stopped positive airway pressure therapy during the 2 years after the start point. Fukurawa et al. [9] addressed adherence to treatment in patients systematically investigated for the presence of OSA when presenting with hypertension. In this population, $17 \%$ of patients having OSA refused to initiate treatment and 43 \% of patients stopped at their own accord after treatment initiation. This particular population showed a higher rate of OSA treatment discontinuation than the control group in our study. This suggests that patients systematically screened for OSA because they have a high cardio-metabolic risk are more likely to stop the treatment than OSA patients screened in a sleep clinic because they present with clinical symptoms of OSA. 
Conversely, in the present study, even though not significant, the apnea-hypopnea index tended to be higher in control patients than in operated patients. There is a possibility that control patients were less likely to abandon OSA treatment because their sleep apnea was more severe leading to an underestimation of discontinuation in controls compared to operated patients.

The proportion of patients stopping OSA treatment following bariatric surgery was greatest between 6 months and 1 year, paralleling the nadir in body weight after surgery. Before and after this period, the rate of discontinuation was not statistically different from that of matched controls. However, our study may be underpowered to capture the difference after 1 year of follow-up.

Factors associated with stopping positive airway pressure treatment after bariatric surgery were female gender, being free

from cardiovascular disease, having a loss in body weight greater than the median of the percent body weight loss observed in this group ($18.4 \%$ ), and having only moderately severe OSA at baseline (under the median AHI, i.e., <35 events/h). On multivariable analysis, none of these factors were independently associated with stopping of treatment, which may indicate that the different factors linked with osA treatment discontinuation in univariate analysis are mutually associated and define a homogenous phenotype of patients who present with higher likelihood of stopping positive airway pressure therapy after bariatric surgery. In meta-analyses of bariatric surgery studies as well as in medical body weight loss studies, the magnitude of body weight loss is recurrently linked to improvement in AHI [22, 29]. This observation refers to the discontinuation of OSA treatment due to authentic recovery. Regarding gender, a recent study by Kennedy-Dalby et al. [30] looked at potential differences in weight loss or metabolic improvement after bariatric surgery in 70 men and 70 matched women. Among them, 80 patients ( 40 men matched to 40 women) presented with OSA treated with CPAP preoperatively. Of these, 31 (77.5 \%) men and 36 (90.0 \%) women stopped CPAP therapy postoperatively. This was for overall OSA treatment discontinuation, whether justified by recovery or not. Prospective and adequately powered studies are needed to better characterize and validate the phenotype of patients who are likely to best benefit from bariatric surgery in terms of OSA resolution, confirmed by post-surgery polysomnography.

\section{Study Limitations}

Our study had several limitations. First, its design was retrospective, preventing us from obtaining some relevant information such as reasons why patients, either controls or operated, stopped their treatment. Thus, the persistent difference observed in discontinuation of OSA treatment between operated patients and controls might either reflect recovery from OSA or an increased dropout after bariatric surgery despite significant persistent OSA. Regarding the control patients, 
over 2 years, we assumed that there was little chance that discontinuation of treatment might have been secondary to OSA recovery, without any etiologic treatment. Thus, we considered that OSA discontinuation in controls was predominantly due to treatment abandon. Another limitation was that the low number of patients followed after 1 year, limited the power of the study in this period.

\section{Conclusion}

The present study confirms that bariatric surgery is associated with a higher rate of stopping positive airway pressure therapy in obese OSA patients compared to carefully matched controls. Female gender, patients with less severe sleep apnea before surgery, freedom from cardiovascular disease, and those with significant post-surgery weight loss could describe the phenotype having a greater likelihood of stopping OSA treatment after surgery.

The true impact of bariatric surgery on treatment discontinuation should be considered in terms of the stopping rate in control obese OSA patients. Previous uncontrolled cohort studies are likely to have overestimated the effect of bariatric surgery on positive airway pressure therapy discontinuation. Further studies might address in which proportion the remaining increase in OSA treatment discontinuation associated with bariatric surgery is justified by objective recovery from OSA and which part consists of increased abandonment by patients considering themselves as cured.

Acknowledgments We thank Nathalie Arnol who performed statistical analyses, Matthieu Lesgoirres who was involved in collecting retrospective data and Dr. Alison Foote (Grenoble Clinical Research Center) for critically reading and editing the manuscript with respect to English language.

\section{Compliance with Ethical Standards}

Grants "Fonds Agir pour les maladies chroniques"

Funding The present study was supported by "Fond Agir pour les maladies chroniques."

Disclosure Summary CA: has no relevant conflict of interest to disclose

JCB: is employed by "Agiràdom", a non-profit home care provider

FR: has nothing to declare regarding this study.

CA: has nothing to declare regarding this study.

NW: has nothing to declare regarding this study. 
SJ: has nothing to declare regarding this study.

DJ: has nothing to declare regarding this study.

JLP: has nothing to declare regarding this study.

ALB: received grant support from "Agiràdom", a non-profit home

care provider in 2009, 2011, 2012, 2013 and 2014

According to French law, this database study using data from routine clinical practice records, did not require informed consent. The study was approved by the institutional ethics committee and has been performed in accordance with the ethical standards as laid down in the 1964 Declaration of Helsinki and its amendments or comparable ethical standards.

\section{References}

1. Young $T$, Peppard PE, Taheri S. Excess weight and sleepdisordered breathing. J Appl Physiol. 2005;99:1592-9.

2. Peppard PE, Young $T$, Palta $M$, et al. Longitudinal study of moderate weight change and sleep-disordered breathing. JAMA.

$2000 ; 284: 3015-21$.

3. Punjabi NM. The epidemiology of adult obstructive sleep apnea. Proc Am Thorac Soc. 2008;5:136-43.

4. Jordan AS, McSharry DG, Malhotra A. Adult obstructive sleep apnoea. Lancet. 2014;383:736-47.

5. Baguet JP, Barone-Rochette G, Tamisier R, Levy P, Pepin JL. Mechanisms of cardiac dysfunction in obstructive sleep apnea. Nat Rev Cardiol. 2012.

6. Pepin JL, Tamisier R, Levy P. Obstructive sleep apnoea and metabolic syndrome: put CPAP efficacy in a more realistic perspective. Thorax. $2012 ; 67: 1025-7$.

7. Meurice JC, Dore P, Paquereau J, et al. Predictive factors of longterm compliance with nasal continuous positive airway pressure treatment in sleep apnea syndrome. Chest. 1994;105:429-33.

8. Kribbs NB, Pack AI, Kline LR, et al. Objective measurement of patterns of nasal CPAP use by patients with obstructive sleep apnea. Am Rev Respir Dis. 1993;147:887-95.

9. Furukawa T, Suzuki M, Ochiai M, et al. Long-term adherence to nasal continuous positive airway pressure therapy by hypertensive patients with preexisting sleep apnea. J Cardiol. 2014;63:281-5. 
10. Pepin JL, Krieger J, Rodenstein D, et al. Effective compliance during the first 3 months of continuous positive airway pressure. A European prospective study of 121 patients. Am J Respir Crit Care Med. $1999 ; 160: 1124-9$.

11. Carneiro G, Florio RT, Zanella MT, et al. Is mandatory screening for obstructive sleep apnea with polysomnography in all severely obese patients indicated? Sleep Breath. 2012;16:163-8.

12. Khan A, King WC, Patterson EJ, et al. Assessment of obstructive sleep apnea in adults undergoing bariatric surgery in the longitudinal assessment of bariatric surgery-2 (LABS-2) study. J Clin Sleep Med. $2013 ; 9: 21-9$.

13. Rao A, Tey BH, Ramalingam G, et al. Obstructive sleep apnoea (OSA) patterns in bariatric surgical practice and response of OSA to weight loss after laparoscopic adjustable gastric banding (LAGB). Ann Acad Med Singap. 2009;38:587-7.

14. Rasmussen JJ, Fuller WD, Ali MR. Sleep apnea syndrome is significantly underdiagnosed in bariatric surgical patients. Surg Obes Relat Dis. 2012;8:569-73.

15. Ravesloot MJ, van Maanen JP, Hilgevoord AA, et al. Obstructive sleep apnea is underrecognized and underdiagnosed in patients undergoing bariatric surgery. Eur Arch Otorhinolaryngol. 2012;269: $1865-71$.

16. Lopes Neto JM, Brandao LO, Loli A, et al. Evaluation of obstructive sleep apnea in obese patients scheduled for bariactric surgery. Acta Cir Bras. 2013;28:317-22.

17. Karason $K$, Lindroos $A K$, Stenlof $K$, et al. Relief of cardiorespiratory symptoms and increased physical activity after surgically induced weight loss: results from the Swedish Obese Subjects study. Arch Intern Med. 2000;160:1797-802.

18. Romero-Corral A, Caples SM, Lopez-Jimenez F, et al. Interactions between obesity and obstructive sleep apnea: implications for treatment. Chest. 2010;137:711-9.

19. Schachter L. Respiratory assessment and management in bariatric surgery. Respirology. 2012;17:1039-47.

20. Buchwald H, Avidor Y, Braunwald E, et al. Bariatric surgery: a systematic review and meta-analysis. JAMA. 2004;292:1724-37.

21. Sarkhosh K, Switzer NJ, El-Hadi M, et al. The impact of bariatric surgery on obstructive sleep apnea: a systematic review. Obes Surg. $2013 ; 23: 414-23$. 
22. Greenburg DL, Lettieri CJ, Eliasson AH. Effects of surgical weight loss on measures of obstructive sleep apnea: a meta-analysis. Am J Med. $2009 ; 122: 535-42$.

23. Ashrafian H, le Roux CW, Rowland SP, et al. Metabolic surgery and obstructive sleep apnoea: the protective effects of bariatric procedures. Thorax. 2012;67:442-9.

24. Borel JC, Tamisier R, Dias-Domingos $S$, et al. Type of mask may impact on continuous positive airway pressure adherence in apneic patients. PLoS One. 2013;8:e64382.

25. Weaver TE, Grunstein RR. Adherence to continuous positive airway pressure therapy: the challenge to effective treatment. Proc Am Thorac Soc. $2008 ; 5: 173-8$.

26. Gagnadoux F, Le Vaillant M, Goupil F, et al. Influence of marital status and employment status on long-term adherence with continuous positive airway pressure in sleep apnea patients. PLos One. 2011;6, e22503.

27. Wozniak DR, Lasserson TJ, Smith I. Educational, supportive and behavioural interventions to improve usage of continuous positive airway pressure machines in adults with obstructive sleep apnoea. Cochrane Database Syst Rev. 2014;1:CD007736.

28. Collen J, Lettieri CJ, Eliasson A. Postoperative CPAP use impacts long-term weight loss following bariatric surgery. J Clin Sleep Med. $2015 ; 11: 213-7$.

29. Alfa Wali M, Ashrafian H, Schofield KL, Harling L, Alkandari A, Darzi A et al. Is social deprivation associated with weight loss outcomes following bariatric surgery? A 10-year single institutional experience. Obes Surg. 2014;24:2126-32.

30. Kennedy-Dalby A, Adam S, Ammori BJ, et al. Weight loss and metabolic outcomes of bariatric surgery in men versus women-a matched comparative observational cohort study. Eur J Intern Med. $2014 ; 25: 922-5$. 
